\title{
Laplace Transform Methods for a Free Boundary Problem of Time-Fractional Partial Differential Equation System
}

\author{
Zhiqiang Zhou and Xuemei Gao \\ School of Economic Mathematics, Southwestern University of Finance and Economics, Wenjiang, Chengdu 611130, China \\ Correspondence should be addressed to Zhiqiang Zhou; zqzhou@2014.swufe.edu.cn
}

Received 28 March 2017; Revised 24 May 2017; Accepted 8 June 2017; Published 10 July 2017

Academic Editor: Mohammad Javidi

Copyright (c) 2017 Zhiqiang Zhou and Xuemei Gao. This is an open access article distributed under the Creative Commons Attribution License, which permits unrestricted use, distribution, and reproduction in any medium, provided the original work is properly cited.

\begin{abstract}
We study the pricing of the American options with fractal transmission system under two-state regime switching models. This pricing problem can be formulated as a free boundary problem of time-fractional partial differential equation (FPDE) system. Firstly, applying Laplace transform to the governing FPDEs with respect to the time variable results in second-order ordinary differential equations (ODEs) with two free boundaries. Then, the solutions of ODEs are expressed in an explicit form. Consequently the early exercise boundaries and the values for the American option are recovered using the Gaver-Stehfest formula. Numerical comparisons of the methods with the finite difference methods are carried out to verify the efficiency of the methods.
\end{abstract}

\section{Introduction}

Markov regime switching models were first introduced by Hamilton [1] and recently have become popular in financial applications including equity options [2-17], bond prices and interest rate derivatives [18-20], portfolio selection [21], and trading rules [22-26]. The Markov regime switching models allow the model parameters (drift and volatility coefficients) to depend on a Markov chain which can reflect the information of the market environments and at the same time preserve the simplicity of the models. However when the model parameters are governed by the Markov chain, the valuation of the options becomes complex.

American option pricing is a kind of classical free boundary problems, where the free boundary refers to early exercise boundary or optimal exercise boundary. Laplace transform methods have been developed to solve the free boundary problems arising in American option pricing under geometric Brownian motion (GBM) (see Mallier and Alobaidi [27], Zhu [28], and Zhu and Zhang [29]) and constant elasticity of variance (CEV) (see Wong and Zhao [30]). The essential idea of the Laplace transform methods for solving the American option pricing problems is described as follows. Applying Laplace transform to the governing free boundary partial differential equations (PDEs) with respect to the time variable results in a boundary value problem of second-order ordinary differential equations (ODEs). Unlike the case for the European option pricing, the ODE involves an unknown boundary (in Laplace space) which needs to be solved. If the solution of the ODE with the unknown boundary can be expressed in an explicit form, then a nonlinear algebraic equation for the unknown boundary can be derived. Using a simple solver for the nonlinear algebraic equation, we can get the value of the unknown boundary in Laplace space. Consequently the early exercise boundary and the value for the American option can be achieved using the inverse Laplace transform.

Chen et al. [31] studied a predictor-corrector finite difference methods for pricing American options under the FMLS model which is a kind of space-fractional derivative model. Liang et al. [32] used Fourier transform to solve a bifractional Black-Scholes-Merton differential equation. In this paper, we study the pricing of American options with time-fractional model which has essential difference to the space-fractional model. Following the model in [33], we assume that the underlying asset price still follows the classical Brownian motion, but the change in the option price is considered as a fractal transmission system. The price of such American 
option follows time-fractional partial differential equations (PDEs) with free boundaries. The solution of the timefractional PDEs with two free boundaries is more challenging than solving the fractional PDEs with fixed boundary for European option pricing in [33] and much more complex than solving single Black-Scholes-Merton differential equation in [32].

In this paper, we develop the Laplace transform methods for pricing time-fractional American options under regime switching models. The fundamental difference to the GBM and CEV American option pricing is that the governing free boundary PDEs for the regime switching American option pricing are a coupled system. Therefore the Laplace transform in time for the system of free boundary PDEs leads to a coupled system of ODEs whose analytic solutions take much trouble to the approach. A careful derivation in different cases makes the approach successful. Numerical examples are provided to verify the efficiency of the approach. The generated option value and early exercise boundary are compared with the finite difference method which are usually used as the benchmark methods in the area of option pricing.

\section{Laplace Transform Methods for American Option Pricing}

Let the underlying asset prices $S_{t}$ follow a two-state regime switching model under risk-neutral measure:

$$
\frac{d S_{t}}{S_{t}}=r(Y(t)) d t+\sigma(Y(t)) d W_{t},
$$

where $W_{t}$ is a standard Brownian motion and $Y(t)$ is a continuous-time Markov chain with two states $\left(y_{1}, y_{2}\right)$. Assume that, at each state $Y(t)=y_{k}, k=1,2$, the interest rates $r\left(y_{k}\right)=r_{k}$ and volatility $\sigma\left(y_{k}\right)=\sigma_{k}$ for $k=1,2$ are nonnegative constants. Let

$$
A=\left[\begin{array}{cc}
-q_{1} & q_{1} \\
q_{2} & -q_{2}
\end{array}\right]
$$

be the generator matrix of the Markov chain process with $q_{i} \geq 0(i=1,2)$. Let $T$ be the option expiration date, $\tau=T-t$ be the time to maturity, $V_{i}(S, \tau)$ represent the American put option prices with stock price $S$, and $\bar{S}_{i}(\tau)$ be the corresponding optimal exercise boundaries at time $\tau$. Then the American put options satisfy the following system of PDEs with free boundaries, for $i=1,2$ :

$$
\frac{\partial V_{i}}{\partial \tau}=L_{i}\left(V_{i}\right)+q_{i} V_{j}, \quad j \neq i, S>\bar{S}_{i}(\tau), \tau>0,
$$

where $L_{i}$ are the differential operators defined by

$$
L_{i}=\frac{1}{2} \sigma_{i}^{2} S^{2} \frac{\partial^{2}}{\partial S^{2}}+r_{i} S \frac{\partial}{\partial S}-\left(r_{i}+q_{i}\right) \text {. }
$$

However, it is argued that the time derivative $\partial V_{i} / \partial \tau$ should be replaced by the fractional derivative $\partial^{\alpha} V_{i} / \partial \tau^{\alpha}(0<\alpha \leq$ 1) under the assumption that the change in the option price follows a fractal transmission system (see, e.g., [3234]). So, the time-fractional American put options satisfy the following system of PDEs:

$$
\frac{\partial V_{i}}{\partial \tau^{\alpha}}=L_{i}\left(V_{i}\right)+q_{i} V_{j}, \quad j \neq i, S>\bar{S}_{i}(\tau), \tau>0,
$$

with the free boundary conditions and the initial condition:

$$
\begin{aligned}
V_{i}(S, \tau) & =K-S, \quad 0 \leq S \leq \bar{S}_{i}(\tau), \\
V_{i}(S, 0) & =\max (K-S, 0), \\
\lim _{S \uparrow \infty} V_{i}(S, \tau) & =0, \\
\lim _{S \downarrow \bar{S}_{i}(\tau)} V_{i}(S, \tau) & =K-\bar{S}_{i}(\tau), \\
\lim _{S \downarrow \bar{S}_{i}(\tau)} \frac{\partial}{\partial S} V_{i}(S, \tau) & =-1,
\end{aligned}
$$

where $\partial V_{i} / \partial \tau^{\alpha}$ is defined as the Caputo fractional derivative for $i=1,2$ :

$$
\begin{aligned}
& \frac{\partial V_{i}}{\partial \tau^{\alpha}} \\
& \quad \equiv \begin{cases}\frac{1}{\Gamma(1-\alpha)} \int_{0}^{\tau} \frac{1}{(\tau-\theta)^{\alpha}} \frac{\partial V_{i}(S, \theta)}{\partial \theta} d \theta, & 0<\alpha<1, \\
\frac{\partial V_{i}}{\partial \tau}, & \alpha=1 .\end{cases}
\end{aligned}
$$

Condition (10) is called "high-contact condition" or "smoothpasting condition."

For $\lambda>0$, we define the Laplace-Carson transform (LCT) of the American put option price $V_{i}(x, \tau)$ as

$$
\begin{aligned}
\widehat{V}_{i}(S, \lambda) & =\int_{0}^{+\infty} V_{i}(S, \tau) \lambda e^{-\lambda \tau} d \tau \\
& =: \mathscr{L}_{\mathscr{C}}\left[V_{i}(S, \tau)\right](\lambda),
\end{aligned}
$$

which is essentially the Laplace transform (LT) as

$$
\mathscr{L}_{\mathscr{C}}\left[V_{i}(S, \tau)\right](\lambda)=\lambda \mathscr{L}\left[V_{i}(S, \tau)\right](\lambda) .
$$

Using the Laplace transform formula for the Caputo fractional derivative (see (2.253) in [35]),

$$
\begin{aligned}
\mathscr{L}\left[\frac{\partial V_{i}(S, \tau)}{\partial \tau^{\alpha}}\right](\lambda)= & \lambda^{\alpha} \mathscr{L}\left[V_{i}(S, \tau)\right](\lambda) \\
& -\lambda^{\alpha-1} V_{i}(S, 0),
\end{aligned}
$$

and the relationship (13), the LCT for $\partial V_{i} / \partial \tau^{\alpha}$ is found as

$$
\begin{aligned}
\mathscr{L}_{\mathscr{C}}\left[\frac{\partial V_{i}(S, \tau)}{\partial \tau^{\alpha}}\right](\lambda)= & \lambda^{\alpha} \mathscr{L}_{\mathscr{C}}\left[V_{i}(S, \tau)\right](\lambda) \\
& -\lambda^{\alpha} V_{i}(S, 0) .
\end{aligned}
$$

The use of LCT simplifies notations in the later analysis and makes inverse Laplace solutions have a unique singularity $\lambda=$ 
0 . We note that for the case $\alpha=1$ formula (15) is still true. Denoting $\widehat{S}_{i}(\lambda)=\mathscr{L} \mathscr{C}\left[\bar{S}_{i}(\tau)\right](\lambda)$ and taking LCTs to (3)-(10), we obtain the following ODEs, for $i=1,2$ :

$$
\begin{aligned}
& \frac{1}{2} \sigma_{i}^{2} S^{2} \frac{\partial^{2} \widehat{V}_{i}}{\partial S^{2}}+r_{i} S \frac{\partial \widehat{V}_{i}}{\partial S}-\left(r_{i}+q_{i}+\lambda^{\alpha}\right) \widehat{V}_{i}+q_{i} \widehat{V}_{j} \\
& \quad+\lambda^{\alpha} \max (K-S, 0)=0, \quad S>\widehat{S}_{i}(\lambda), \\
& \widehat{V}_{i}(S, \lambda)=K-S, \quad 0 \leq S \leq \widehat{S}_{i}(\lambda), \\
& \lim _{S \uparrow \infty} \widehat{V}_{i}(S, \lambda)=0, \\
& \lim _{S \downarrow \widehat{S}_{i}(\lambda)} \widehat{V}_{i}(S, \lambda)=K-\widehat{S}_{i}(\lambda), \\
& \lim _{S \downarrow \widehat{S}_{i}(\lambda)} \frac{\partial}{\partial S} \widehat{V}_{i}(S, \lambda)=-1 .
\end{aligned}
$$

The existence of Laplace transform $\widehat{S}_{i}(\lambda)$ and $\widehat{V}_{i}(S, \lambda)$ is guaranteed by the continuity and boundedness of $\bar{S}_{i}(\tau)$ and $V_{i}(S, \tau)$. To solve (16)-(20), we use the following variable and function transformations:

$$
\begin{aligned}
x & =\ln \frac{S}{K}, \\
\widehat{x}_{i}(\lambda) & =\ln \frac{\widehat{S}_{i}(\lambda)}{K}, \\
\widehat{V}_{i}(S, \lambda) & =K \widehat{U}_{i}(x, \lambda) .
\end{aligned}
$$

Let $a_{i}=(1 / 2) \sigma_{i}^{2}, b_{i}=r_{i}-(1 / 2) \sigma_{i}^{2}, c_{i}=r_{i}+q_{i}$. Then the calculations yield an equivalent form to (16)-(20) as follows:

$$
\begin{aligned}
& a_{i} \frac{\partial^{2} \widehat{U}_{i}}{\partial x^{2}}+b_{i} \frac{\partial \widehat{U}_{i}}{\partial x}-\left(c_{i}+\lambda^{\alpha}\right) \widehat{U}_{i}+q_{i} \widehat{U}_{j} \\
& \quad+\lambda^{\alpha} \max \left(1-e^{x}, 0\right)=0, \quad x>\widehat{x}_{i}(\lambda), \\
& \widehat{U}_{i}(x, \lambda)=1-e^{x}, \quad-\infty \leq x \leq \widehat{x}_{i}(\lambda), \\
& \lim _{x \uparrow \infty} \widehat{U}_{i}(x, \lambda)=0, \\
& \lim _{x \downarrow \widehat{x}_{i}(\lambda)} \widehat{U}_{i}(x, \lambda)=1-e^{\widehat{x}_{i}(\lambda)}, \\
& \lim _{x \downarrow \widehat{x}_{i}(\lambda)} \frac{\partial}{\partial x} \widehat{U}_{i}(x, \lambda)=-e^{\widehat{x}_{i}(\lambda)} .
\end{aligned}
$$

Compared to (16), the coefficients of the first and second derivatives in (22) become constant which will simplify the calculation. However, (22) includes two unknown variables $\widehat{x}_{i}(i=1,2)$ and a nonsmooth term $\max \left(1-e^{x}, 0\right)$, which will make the solutions somewhat complicated.

From the definition of $\widehat{S}_{i}(\lambda)$ and monotone decreasing property of $\bar{S}_{i}(\tau)$ (see, e.g., [10]), we have

$$
0<\widehat{S}_{i}(\lambda)=\mathscr{L} \mathscr{C}[\bar{S}(\tau)](\lambda)<\bar{S}_{i}(0)=K,
$$

and therefore

$$
-\infty<\widehat{x}_{i}(\lambda)=\ln \left(\frac{\widehat{S}_{i}(\lambda)}{K}\right)<0 .
$$

Moreover $\widehat{U}_{i}(x, \lambda)$ and its first-order derivative are continuous at $x=0\left(x=0\right.$ means that $\left.\widehat{S}_{i}=K\right)$. Without loss of generality, we assume $\widehat{x}_{1}(\lambda)<\widehat{x}_{2}(\lambda)$. So $-\infty<\widehat{x}_{1}(\lambda)<$ $\widehat{x}_{2}(\lambda)<0$. The solutions of $(22)-(26)$ can be solved separately on four intervals $I_{1}=\left(-\infty, \widehat{x}_{1}\right), I_{2}=\left[\widehat{x}_{1}, \widehat{x}_{2}\right), I_{3}=\left[\widehat{x}_{2}, 0\right)$, and $I_{4}=[0,+\infty)$.

Proposition 1. The solutions of (22)-(26) on interval $I_{1}=$ $\left(-\infty, \widehat{x}_{1}\right)$ are given by

$$
\begin{aligned}
& \widehat{U}_{1}(x, \lambda)=1-e^{x}, \\
& \widehat{U}_{2}(x, \lambda)=1-e^{x},
\end{aligned}
$$

$$
x \in I_{1} .
$$

Proof. The proof is straightforward by noticing (23).

The solutions of ODEs on the other intervals can be written as a general solution to the corresponding homogeneous part plus a particular one satisfying the nonhomogeneous system. More precisely, we discuss the solutions on the different intervals as follows.

Proposition 2. On interval $I_{2}=\left[\widehat{x}_{1}, \widehat{x}_{2}\right)$, the solutions of (22)-(26) are given by

$$
\begin{aligned}
& \widehat{U}_{1}(x, \lambda)=\boldsymbol{\Phi}(x) \mathbf{C}_{1}+\mu_{\lambda}(x), \\
& \widehat{U}_{2}(x, \lambda)=1-e^{x},
\end{aligned}
$$

$$
x \in I_{2},
$$

and constant $\mathbf{C}_{1}$ is uniquely determined by the boundary conditions (25) and (26); that is,

$$
\mathrm{C}_{1}=\widetilde{\boldsymbol{\Phi}}^{-1}\left(\widehat{x}_{1}\right) \mathbf{f}_{1}\left(\widehat{x}_{1}\right) \text {. }
$$

Here the unspecified notations are defined in (34)-(37) below.

Proof. For $x \in I_{2}$, the solution $\widehat{U}_{2}(x, \lambda)=1-e^{x}$ and then $\widehat{U}_{1}(x, \lambda)$ satisfies the following equation:

$$
\begin{aligned}
& a_{1} \frac{\partial^{2} \widehat{U}_{1}}{\partial x^{2}}+b_{1} \frac{\partial \widehat{U}_{1}}{\partial x}-\left(c_{1}+\lambda^{\alpha}\right) \widehat{U}_{1}+\left(q_{1}+\lambda^{\alpha}\right)\left(1-e^{x}\right) \\
& \quad=0 .
\end{aligned}
$$

By solving ODE (32), we get the general solution

$$
\widehat{U}_{1}(x, \lambda)=C_{11} \phi_{1}(x)+C_{12} \phi_{2}(x)+\mu_{\lambda}(x),
$$

where $\mu_{\lambda}(x)$ is a particular solution and $\phi_{1}(x)$ and $\phi_{2}(x)$ are the fundamental increasing and decreasing solutions with explicit forms:

$$
\begin{aligned}
\mu_{\lambda}(x) & =\frac{q_{1}+\lambda^{\alpha}}{c_{1}+\lambda^{\alpha}}+\frac{q_{1}+\lambda^{\alpha}}{a_{1}+b_{1}-c_{1}-\lambda^{\alpha}} e^{x}, \\
\phi_{1}(x) & =e^{\gamma_{1}^{+} x}, \\
\phi_{2}(x) & =e^{\gamma_{1}^{-} x}, \\
\gamma_{1}^{ \pm} & =\frac{-b_{1} \pm \sqrt{b_{1}^{2}+4 a_{1}\left(c_{1}+\lambda^{\alpha}\right)}}{2 a_{1}} .
\end{aligned}
$$


Note that $\gamma_{1}^{+}>0$ and $\gamma_{1}^{-}<0$. Denoting

$$
\begin{aligned}
\mathbf{C}_{1} & =\left[\begin{array}{l}
C_{11} \\
C_{12}
\end{array}\right], \\
\boldsymbol{\Phi}(x) & =\left[\begin{array}{l}
\phi_{1}(x) \\
\phi_{2}(x)
\end{array}\right]^{\mathrm{T}}, \\
\widetilde{\boldsymbol{\mu}}_{\lambda}(x) & =\left[\begin{array}{c}
\mu_{\lambda}(x) \\
\frac{d}{d x} \mu_{\lambda}(x)
\end{array}\right], \\
\widetilde{\boldsymbol{\Phi}}(x) & =\left[\begin{array}{c}
\boldsymbol{\Phi}(\mathbf{x}) \\
\frac{d}{d x} \mathbf{\Phi}(\mathbf{x})
\end{array}\right], \\
\mathbf{f}_{1}(x) & =\left[\begin{array}{c}
1-e^{x} \\
-e^{x}
\end{array}\right]-\widetilde{\boldsymbol{\mu}}_{\lambda}(x),
\end{aligned}
$$

and using (23), we complete the proof.

Now we derive the solutions of (22)-(26) on interval $I_{3}=$ $\left[\widehat{x}_{2}, 0\right)$. Let $\mathbf{y}(x)=\left[y_{1}(x), y_{2}(x), y_{3}(x), y_{4}(x)\right]^{\mathrm{T}}$ with

$$
\begin{aligned}
& y_{1}(x)=\widehat{U}_{1}(x, \lambda), \\
& y_{2}(x)=\frac{\partial \widehat{U}_{1}(x, \lambda)}{\partial x}, \\
& y_{3}(x)=\widehat{U}_{2}(x, \lambda), \\
& y_{4}(x)=\frac{\partial \widehat{U}_{2}(x, \lambda)}{\partial x} .
\end{aligned}
$$

Then (22) is equivalent to the following first-order ODE system:

$$
\mathbf{y}^{\prime}(x)=\mathbf{A y}(x)+\mathbf{g}(x)
$$

where

$$
\begin{aligned}
\mathbf{A} & =\left[\begin{array}{cccc}
0 & 1 & 0 & 0 \\
\frac{c_{1}+\lambda^{\alpha}}{a_{1}} & -\frac{b_{1}}{a_{1}} & -\frac{q_{1}}{a_{1}} & 0 \\
0 & 0 & 0 & 1 \\
-\frac{q_{2}}{a_{2}} & 0 & \frac{c_{2}+\lambda^{\alpha}}{a_{2}} & -\frac{b_{2}}{a_{2}}
\end{array}\right], \\
\mathbf{g}(x) & =\left[\begin{array}{c}
\lambda^{\alpha}\left(e^{x}-1\right) \\
0 \\
\lambda^{\alpha}\left(e^{x}-1\right)
\end{array}\right] .
\end{aligned}
$$

Lemma 3. For any real number $\lambda>0$, the homogeneous equation $\mathbf{y}^{\prime}(x)=\mathbf{A y}(x)$ has real valued fundamental solutions

$$
\widetilde{\boldsymbol{\Psi}}(x)=\left[e^{\nu_{1} x} \xi_{1}, e^{\nu_{2} x} \xi_{2}, e^{\nu_{3} x} \xi_{3}, e^{\nu_{4} x} \boldsymbol{\xi}_{4}\right],
$$

where $v_{1}$ and $v_{2}$ are different negative real numbers, $v_{3}$ and $v_{4}$ are different positive real numbers, and $\boldsymbol{\xi}_{\mathbf{j}}(j=1,2,3,4)$ are real valued column vectors.

Proof. It is well-known that $\mathbf{y}^{\prime}(x)=\mathbf{A y}(x)$ has fundamental solution matrix as (41), with $v_{j}$ and $\xi_{\mathbf{j}}$ representing characteristic values and corresponding characteristic vectors. So we only need to prove all $v_{j}$ are real numbers and different from each other. The characteristic polynomial of (39) is

$$
\begin{aligned}
P(\nu)= & \operatorname{det}(\nu I-A) \\
= & \left|\begin{array}{cccc}
v & -1 & 0 & 0 \\
-\frac{c_{1}+\lambda^{\alpha}}{a_{1}} & v+\frac{b_{1}}{a_{1}} & \frac{q_{1}}{a_{1}} & 0 \\
0 & 0 & v & -1 \\
\frac{q_{2}}{a_{2}} & 0 & -\frac{c_{2}+\lambda^{\alpha}}{a_{2}} & v+\frac{b_{2}}{a_{2}}
\end{array}\right| \\
= & \left(v^{2}+\frac{b_{1}}{a_{1}} v-\frac{c_{1}+\lambda^{\alpha}}{a_{1}}\right)\left(v^{2}+\frac{b_{2}}{a_{2}} v-\frac{c_{2}+\lambda^{\alpha}}{a_{2}}\right) \\
& -\frac{q_{1} q_{2}}{a_{1} a_{2}} .
\end{aligned}
$$

Therefore,

$$
\begin{aligned}
& P(\nu)=\frac{1}{a_{1} a_{2}}\left\{\left[a_{1} \nu^{2}+b_{1} v-\left(c_{1}+\lambda^{\alpha}\right)\right]\right. \\
& \left.\cdot\left[a_{2} v^{2}+b_{2} v-\left(c_{2}+\lambda^{\alpha}\right)\right]-q_{1} q_{2}\right\} \\
& =\frac{1}{a_{1} a_{2}}\left[\left(\nu-\gamma_{1}^{-}\right)\left(\nu-\gamma_{1}^{+}\right)\left(\nu-\gamma_{2}^{-}\right)\left(\nu-\gamma_{2}^{+}\right)\right. \\
& \left.-q_{1} q_{2}\right]
\end{aligned}
$$

where

$$
\gamma_{i}^{ \pm}=\frac{-b_{i} \pm \sqrt{b_{i}^{2}+4 a_{i}\left(c_{i}+\lambda^{\alpha}\right)}}{2 a_{i}} .
$$

Since $a_{i}=\sigma_{i} / 2>0$ and $c_{i}=r_{i}+q_{i}>0$, for any $\lambda \geq 0$ we know that all $\gamma_{i}^{ \pm}$are real numbers and $\gamma_{i}^{-}<0(i=1,2)$ and $\gamma_{i}^{+}>0(i=1,2)$. Moreover, we have

$$
\begin{aligned}
P\left(\gamma_{i}^{ \pm}\right) & =-\frac{q_{1} q_{2}}{a_{1} a_{2}}<0, \quad i=1,2, \\
P(0) & =\frac{\left(c_{1}+\lambda^{\alpha}\right)\left(c_{2}+\lambda^{\alpha}\right)-q_{1} q_{2}}{a_{1} a_{2}}>0, \\
\lim _{\nu \rightarrow \pm \infty} P(\nu) & >0 .
\end{aligned}
$$

Then there must exist four real roots $v_{j}(j=1,2,3,4)$ solving characteristic equation $P(\nu)=0$, and we can list the ranges of these values:

$$
-\infty<v_{1}<\min _{i}\left\{\gamma_{i}^{-}\right\},
$$

$$
\max _{i}\left\{\gamma_{i}^{-}\right\}<v_{2}<0 \text {, }
$$




$$
\begin{aligned}
0 & <v_{3}<\min _{i}\left\{\gamma_{i}^{+}\right\}, \\
\max _{i}\left\{\gamma_{i}^{+}\right\} & <v_{4}<+\infty .
\end{aligned}
$$

Note that the roots $v_{j}(j=1,2,3,4)$ can be searched by the secant method on their corresponding ranges as above.

Proposition 4. Let $\Psi(x)$ be a submatrix extracting the first and the third rows from $\widetilde{\Psi}(x)$. Then the solutions $\widehat{\mathbf{U}}(x, \lambda)=$ $\left(\widehat{U}_{1}, \widehat{U}_{2}\right)^{\mathrm{T}}$ of $(22)-(26)$ on interval $I_{3}=\left[\widehat{x}_{2}(\lambda), 0\right)$ are given by

$$
\widehat{\mathbf{U}}(x, \lambda)=\boldsymbol{\Psi}(x) \mathbf{C}_{2}+\boldsymbol{\eta}_{\lambda}(x),
$$

where $\widetilde{\Psi}(x)$ is given by (41) and $\mathbf{C}_{2}$ by (52) below.

Proof. It can be verified that (22) has a particular solution

$$
\begin{aligned}
\boldsymbol{\eta}_{\lambda}(x)= & {\left[\begin{array}{l}
\eta_{1, \lambda}(x) \\
\eta_{2, \lambda}(x)
\end{array}\right] } \\
= & {\left[\begin{array}{cc}
-\left(c_{1}+\lambda^{\alpha}\right) & q_{1} \\
q_{2} & -\left(c_{2}+\lambda^{\alpha}\right)
\end{array}\right]^{-1}\left[\begin{array}{c}
-\lambda^{\alpha} \\
-\lambda^{\alpha}
\end{array}\right] } \\
& +\left[\begin{array}{ll}
\delta_{1} & q_{1} \\
q_{2} & \delta_{2}
\end{array}\right]^{-1}\left[\begin{array}{l}
\lambda^{\alpha} e^{x} \\
\lambda^{\alpha} e^{x}
\end{array}\right] .
\end{aligned}
$$

Here, $\delta_{i}=a_{i}+b_{i}-\left(c_{i}+\lambda\right)=-q_{i}-\lambda$ for $i=1,2$. Denote $\mathbf{C}_{2}=\left[C_{21}, C_{22}, C_{23}, C_{24}\right]^{\mathrm{T}}$. Then the solution to (39) can be written as

$$
\begin{aligned}
& \mathbf{y}(x)=\widetilde{\boldsymbol{\Psi}}(x) \mathbf{C}_{2}+\widetilde{\boldsymbol{\eta}}_{\lambda}(x), \\
& \widetilde{\boldsymbol{\eta}}_{\lambda}(x) \\
& \quad=\left[\eta_{1, \lambda}(x), \frac{d}{d x} \eta_{1, \lambda}(x), \eta_{2, \lambda}(x), \frac{d}{d x} \eta_{2, \lambda}(x)\right]^{\mathrm{T}} .
\end{aligned}
$$

Using the continuity and smoothing-pasting of solutions at $x=\widehat{x}_{2}$, we know that $\mathbf{C}_{2}$ satisfies

$$
\widetilde{\boldsymbol{\Psi}}\left(\widehat{x}_{2}\right) \mathbf{C}_{2}+\widetilde{\boldsymbol{\eta}}_{\lambda}\left(\widehat{x}_{2}\right)=\left[\begin{array}{c}
\widetilde{\boldsymbol{\Phi}}\left(\hat{x}_{2}\right) \mathbf{C}_{1}+\widetilde{\boldsymbol{\mu}}_{\lambda}\left(\widehat{x}_{2}\right) \\
1-e^{\widehat{x}_{2}} \\
-e^{\widehat{x}_{2}}
\end{array}\right]
$$

Substituting $\mathbf{C}_{1}=\widetilde{\boldsymbol{\Phi}}^{-1}\left(\widehat{x}_{1}\right) \mathbf{f}_{1}\left(\widehat{x}_{1}\right)$ into the above equation and denoting

$$
\begin{aligned}
\mathbf{f}_{2}\left(\widehat{x}_{1}, \widehat{x}_{2}\right)= & {\left[\begin{array}{c}
\widetilde{\boldsymbol{\Phi}}\left(\hat{x}_{2}\right) \widetilde{\boldsymbol{\Phi}}^{-1}\left(\widehat{x}_{1}\right) \mathbf{f}_{1}\left(\widehat{x}_{1}\right)+\widetilde{\boldsymbol{\mu}}_{\lambda}\left(\widehat{x}_{2}\right) \\
1-e^{\widehat{x}_{2}} \\
\\
-e^{\widehat{x}_{2}}
\end{array}\right] } \\
& -\widetilde{\boldsymbol{\eta}}_{\lambda}\left(\widehat{x}_{2}\right),
\end{aligned}
$$

we obtain that

$$
\mathrm{C}_{2}=\widetilde{\boldsymbol{\Psi}}^{-1}\left(\widehat{x}_{2}\right) \mathbf{f}_{2}\left(\widehat{x}_{1}, \widehat{x}_{2}\right) .
$$

Thus the proof is complete.
Proposition 5. Let $\widehat{\Psi}(x)$ be the submatrix extracting the first and the second columns from $\Psi(x)$, where $\Psi(x)$ is given in Proposition 4. Then the solutions $\widehat{\mathbf{U}}(x, \lambda)=\left(\widehat{U}_{1}, \widehat{U}_{2}\right)^{\mathrm{T}}$ of (22)-(26) on interval $I_{4}=[0,+\infty)$ are given by

$$
\widehat{\mathbf{U}}(x)=\widehat{\Psi}(x) \mathbf{C}_{3} .
$$

with $\mathbf{C}_{3}$ given by (56).

Proof. Since $\max \left(1-e^{x}, 0\right)=0$ for $x \in I_{4}=[0,+\infty)$, (22) becomes homogeneous. Furthermore using condition $\lim _{x \uparrow \infty} \widehat{U}_{i}(x, \lambda)=0$ (see $\left.(24)\right)$, we can write the solution to the homogeneous part of (24) (or homogeneous part of (39)) as

$$
\widehat{\mathbf{U}}(x)=\widehat{\Psi}(x) \mathbf{C}_{3},
$$

where $\widehat{\Psi}(x)$ is the submatrix containing the first and the second columns of $\Psi(x)$ and $\mathbf{C}_{3}=\left[C_{31}, C_{32}\right]^{\mathrm{T}}$. Notations $\widetilde{\boldsymbol{\Psi}}, \boldsymbol{\Psi}$, and $\widehat{\boldsymbol{\Psi}}$ are $4 \times 4,2 \times 4$, and $2 \times 2$ matrices, respectively. Since the first-order derivative of $\widehat{U}_{i}(x, \lambda)$ is also continuous at $x=0$, by evaluating the first-order derivatives of (53) and (47) at $x=0$, we obtain that

$$
\Psi^{\prime}(0) \mathbf{C}_{2}+\boldsymbol{\eta}_{\lambda}^{\prime}(0)=\widehat{\Psi}^{\prime}(0) \mathbf{C}_{3},
$$

where $\Psi^{\prime}(0)=\left.(d \Psi(x) / d x)\right|_{x=0}$ and $\widehat{\Psi}^{\prime}(0)=\left.(d \widehat{\Psi}(x) / d x)\right|_{x=0}$. Therefore, we obtain

$$
\mathbf{C}_{3}=\left[\widehat{\Psi}^{\prime}(0)\right]^{-1}\left[\boldsymbol{\Psi}^{\prime}(0) \mathbf{C}_{2}+\boldsymbol{\eta}_{\lambda}^{\prime}(0)\right]
$$

Since $\widehat{U}_{i}(x, \lambda)$ is continuous at $x=0$, by evaluating (53) and (47) at $x=0$, we have

$$
\Psi(0) \mathbf{C}_{2}+\boldsymbol{\eta}_{\lambda}(0)=\widehat{\Psi}(0) \mathbf{C}_{3}
$$

Substituting (52) and (56) into (57) and simplifying the calculation, we obtain a system of nonlinear algebra equations with respect to $\widehat{x}_{1}$ and $\widehat{x}_{2}$

$$
\mathbf{P}_{\lambda} \widetilde{\boldsymbol{\Psi}}^{-1}\left(\widehat{x}_{2}\right) \mathbf{f}_{2}\left(\widehat{x}_{1}, \widehat{x}_{2}\right)+\mathbf{Q}_{\lambda}=0
$$

where constant matrices $\mathbf{P}_{\lambda}$ and $\mathbf{Q}_{\lambda}$ are defined by

$$
\begin{aligned}
& \mathbf{P}_{\lambda}=\widehat{\Psi}(0)\left[\widehat{\Psi}^{\prime}(0)\right]^{-1} \boldsymbol{\Psi}^{\prime}(0)-\boldsymbol{\Psi}(0) \\
& \mathbf{Q}_{\lambda}=\widehat{\Psi}(0)\left[\widehat{\Psi}^{\prime}(0)\right]^{-1} \boldsymbol{\eta}_{\lambda}^{\prime}(0)-\boldsymbol{\eta}_{\lambda}(0)
\end{aligned}
$$

We summarize the above discussions in the following theorem.

Theorem 6. For any fixed $\lambda>0$, the optimal exercise boundaries $\widehat{x}_{1}(\lambda)$ and $\hat{x}_{2}(\lambda)$ can be numerically computed 
TABLE 1: Prices of American put option with parameters $\sigma_{1}=0.8, \sigma_{2}=0.3, r_{1}=0.1, r_{2}=0.05, q_{1}=6, q_{2}=9, K=9, T=1$ and different values of $\alpha$.

\begin{tabular}{|c|c|c|c|c|c|c|c|}
\hline \multirow{2}{*}{$\alpha$} & \multirow{2}{*}{$S_{0}$} & \multicolumn{3}{|c|}{ Regime 1} & \multicolumn{3}{|c|}{ Regime 2} \\
\hline & & LTM & FDM & RE (\%) & LTM & FDM & $\mathrm{RE}(\%)$ \\
\hline \multirow{7}{*}{$\alpha=1$} & 3.00 & 6.0000 & 6.0000 & 0.0000 & 6.0000 & 6.0000 & 0.0000 \\
\hline & 4.50 & 4.4924 & 4.5431 & 1.1160 & 4.5278 & 4.5117 & 0.3568 \\
\hline & 6.00 & 3.3692 & 3.4138 & 1.3065 & 3.3071 & 3.3502 & 1.2865 \\
\hline & 7.50 & 2.5536 & 2.5835 & 1.1573 & 2.4741 & 2.5026 & 1.1388 \\
\hline & 9.00 & 1.9512 & 1.9712 & 1.0146 & 1.8630 & 1.8816 & 0.9885 \\
\hline & 10.50 & 1.5047 & 1.5177 & 0.8566 & 1.4146 & 1.4265 & 0.8342 \\
\hline & 12.00 & 1.1717 & 1.1795 & 0.6613 & 1.0847 & 1.0915 & 0.6230 \\
\hline \multirow{7}{*}{$\alpha=0.7$} & 3.00 & 6.0000 & 6.0000 & 0.0000 & 6.0000 & 6.0000 & 0.0000 \\
\hline & 4.50 & 4.5084 & 4.5436 & 0.7747 & 4.5181 & 4.5149 & 0.0709 \\
\hline & 6.00 & 3.3787 & 3.3988 & 0.5914 & 3.3229 & 3.3420 & 0.5715 \\
\hline & 7.50 & 2.5258 & 2.5381 & 0.4846 & 2.4442 & 2.4537 & 0.3872 \\
\hline & 9.00 & 1.9016 & 1.9089 & 0.3824 & 1.7972 & 1.8037 & 0.3604 \\
\hline & 10.50 & 1.4633 & 1.4672 & 0.2658 & 1.3659 & 1.3691 & 0.2337 \\
\hline & 12.00 & 1.1517 & 1.1531 & 0.1214 & 1.0668 & 1.0678 & 0.0937 \\
\hline \multirow{7}{*}{$\alpha=0.4$} & 3.00 & 6.0000 & 6.0000 & 0.0000 & 6.0000 & 6.0000 & 0.0000 \\
\hline & 4.50 & 4.5275 & 4.5370 & 0.2094 & 4.5067 & 4.5112 & 0.0998 \\
\hline & 6.00 & 3.3630 & 3.3677 & 0.1396 & 3.3102 & 3.3146 & 0.1327 \\
\hline & 7.50 & 2.4738 & 2.4749 & 0.0444 & 2.3871 & 2.3877 & 0.0251 \\
\hline & 9.00 & 1.8271 & 1.8257 & 0.0767 & 1.7102 & 1.7085 & 0.0995 \\
\hline & 10.50 & 1.3915 & 1.3883 & 0.2305 & 1.2882 & 1.2849 & 0.2568 \\
\hline & 12.00 & 1.0940 & 1.0891 & 0.4499 & 1.0094 & 1.0046 & 0.4778 \\
\hline CPU time & \multicolumn{7}{|c|}{$1.98 \mathrm{~s}$ for LTM; $532.47 \mathrm{~s}$ for FDM } \\
\hline
\end{tabular}

from nonlinear equations (58) and the solutions $\widehat{\mathrm{U}}(x, \lambda)=$ $\left[\widehat{U}_{1}(x, \lambda), \widehat{U}_{2}(x, \lambda)\right]^{\mathrm{T}}$ of $(22)-(26)$ are given by

$$
\begin{aligned}
& \widehat{U}_{1}(x, \lambda)=1-e^{x}, \quad x \in I_{1}, \\
& \widehat{U}_{1}(x, \lambda)=\Phi(x) \mathbf{C}_{1}+\mu_{\lambda}(x), \quad x \in I_{2}, \\
& \widehat{U}_{2}(x, \lambda)=1-e^{x}, \quad x \in I_{1} \cup I_{2}, \\
& \widehat{\mathbf{U}}(x, \lambda)=\Psi(x) \mathbf{C}_{2}+\eta_{\lambda}(x), \quad x \in I_{3}, \\
& \widehat{\mathbf{U}}(x, \lambda)=\widehat{\Psi}(x) \mathbf{C}_{3}, \quad x \in I_{4},
\end{aligned}
$$

where $\mathbf{C}_{1}, \mathbf{C}_{2}$, and $\mathbf{C}_{3}$ are given by formulas (31), (52), and (56), respectively; the fundamental solution matrices $\Phi(x)$, $\Psi(x)$, and $\widehat{\Psi}(x)$ are defined in (37) and Propositions 4 and 5, respectively; the particular solutions $\mu_{\lambda}(x)$ and $\boldsymbol{\eta}_{\lambda}(x)$ are given by (34) and (48), respectively.

Finally, the original optimal exercise boundaries $\bar{S}_{i}(\tau)$ and American put option prices $V_{i}(S, \tau)$ can be expressed in terms of the Laplace inversion:

$$
\begin{aligned}
\bar{S}_{i}(\tau) & =K e^{\mathscr{L}^{-1}\left[\widehat{x}_{i}(\lambda) / \lambda\right]}, \quad i=1,2, \\
V_{i}(S, \tau) & =K \mathscr{L}^{-1}\left[\frac{1}{\lambda} \widehat{U}_{i}\left(\ln \frac{S}{K}, \lambda\right)\right], \quad i=1,2 .
\end{aligned}
$$

The numerical approximation for Laplace inversion $f(t)=\mathscr{L}^{-1} \hat{f}(\lambda)$ can be computed using the most powerful Gaver-Stehfest algorithm (see, e.g., [36])

$$
f(t)=\frac{\ln 2}{t} \sum_{k=1}^{n} C_{k}^{(n)} \hat{f}\left(\frac{k \ln 2}{t}\right),
$$

with

$$
\begin{aligned}
C_{k}^{(n)} & =(-1)^{k+n / 2} \\
& \cdot \sum_{j=[(k+1) / 2]}^{\min [k, n / 2]} \frac{j^{n / 2}(2 j) !}{(n / 2-j) ! j !(j-1) !(k-j) !(2 j-k) !} ;
\end{aligned}
$$

here $n$ must be taken as even number.

\section{Numerical Examples}

In this section, the Laplace transform method (LTM) is compared with the finite difference method (FDM). We use symbolic manipulation and multiprecision computing provided by Mathematica 9.0.

Table 1 lists the computational values of American put options under regime switching. Columns entitled "LTM" and "FDM" represent the option values obtained by Laplace transform method and finite different method, respectively. For the LTM the Gaver-Stehfest formula is applied with the number $n=6$ and for the FDM the number of time 


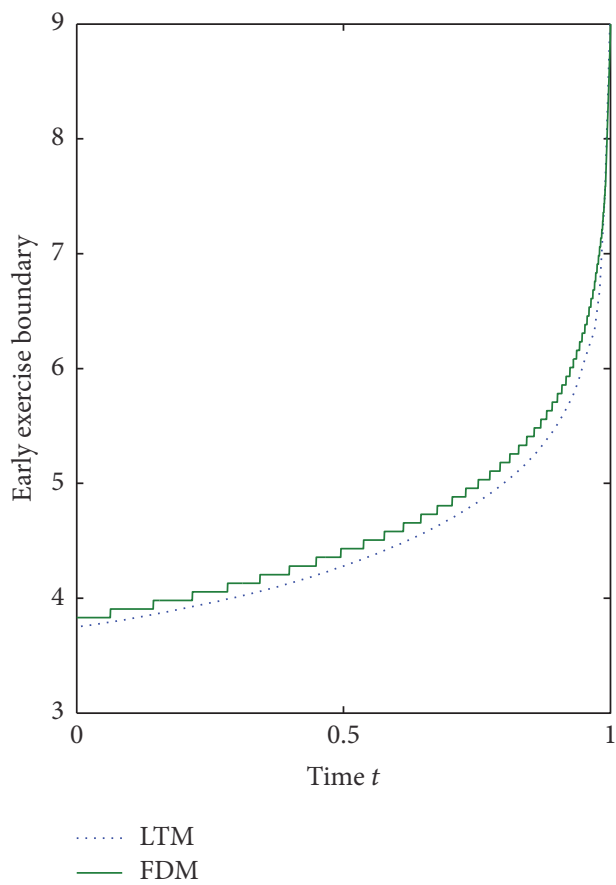

(a)

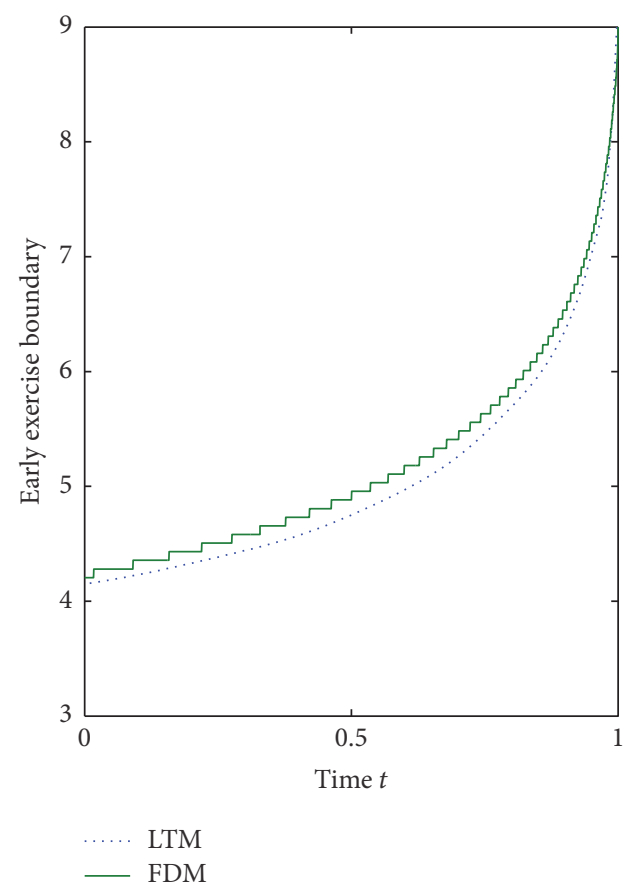

(b)

FIGURE 1: Early exercise boundaries computed by LTM and FDM with $\alpha=0.7$ and the same parameters as in Table 1 . (a) is for regime 1 and (b) for regime 2 .

nodes $M=200$ and the number of space nodes $N=$ 400. The relative error (RE) is the absolute value of the difference between the computed LTM option values and the FDM values divided by the FDM option values. In the implementation of the LTM, the work precision is set by 30 significant digits, while the numerical values are presented with 5 significant digits.

The relative errors shown in Table 1 are less than $1.5 \%$ for all the cases, which illustrates that the LTM is competitively accurate for solving American option pricing problems. Since there are no exact solutions of FPDEs, we cannot judge which one of the two results is more accurate. In general, the relative error less than five percent is acceptable in financial engineering practice.

From the last row of Table 1, we see that the CPU time of LTM is much less than that of FDM. The computational cost of LTM is uniform at every time $t=T-\tau$ and the CPU consumption mainly comes from solving $n$ nonlinear algebraic equations with two variables $\widehat{x}_{1}$ and $\widehat{x}_{2}$ (see expression (58)). FDM is a time-stepping scheme which is needed to solve $M$ nonlinear systems at time $\tau=T$ or $t=0$. Moreover, for the global dependence of the fractional differential operator, FDM needs to do some additional computation. We observe that the numbers $n=6$ of the quadrature nodes are much smaller than the numbers $M=200$ of time steps. In other words, to achieve the similar accuracy, there are much fewer nonlinear systems needed to be solved by the LTM than that by the FDM.

Figure 1 plots the early exercise boundaries obtained by LTM and FDM with the same values of parameters as in
Table 1. It can be seen that the boundaries computed by FDM and LTM are pretty close. Again it shows that the LTM is robust in generating the early exercise boundaries.

\section{Conclusions}

In this paper, we have developed Laplace transform methods to solve the time-fractional American option pricing under regime switching models. The value of the American option with regime switching is formulated as the solution to a free boundary problem of time-fractional partial differential equation system. The Laplace transform is executed for the time variables and the resulting system PDEs are solved analytically. Consequently a system of nonlinear algebraic equations for the free boundaries is obtained and solved using secant methods. Finally numerical Laplace inversion is applied to recover the early exercise boundaries and the option values. Comparisons between the LTM and the benchmark FDM are made via numerical examples, which shows that the LTM is efficient for pricing American options with regime switching. However, the LTM is still challenging for more complex models like the regime switching models with state-dependent jump diffusions. This will be left for the future studies.

\section{Nomenclature of Notations}
$S:$ Stock price
$r_{i}$ : Interest rate
$\sigma_{i}$ : Volatility 
$\bar{S}_{i}$ : Free boundary

$K$ : Strike price

A: Generator matrix of the Markov chain process

$\alpha$ : Order of the Caputo derivative

$V_{i}$ : Option price.

\section{Conflicts of Interest}

The authors declare that there are no conflicts of interest regarding the publication of this paper.

\section{References}

[1] J. D. Hamilton, "A new approach to the economic analysis of nonstationary time series and the business cycle," Econometrica, vol. 57, no. 2, pp. 357-384, 1989.

[2] D. D. Aingworth, S. R. Das, and R. Motwani, "A simple approach for pricing equity options with Markov switching state variables," Quantitative Finance, vol. 6, no. 2, pp. 95-105, 2006.

[3] N. P. B. Bollen, "Valuing options in regime-switching models," The Journal of Derivatives, vol. 6, no. 1, pp. 38-49, 1998.

[4] P. P. Boyle and T. Draviam, "Pricing exotic options under regime switching," Insurance: Mathematics and Economics, vol. 40, no. 2, pp. 267-282, 2007.

[5] J. Buffington and R. J. Elliott, "American options with regime switching," International Journal of Theoretical and Applied Finance, vol. 5, no. 5, pp. 497-514, 2002.

[6] P. Eloe, R. H. Liu, and J. Y. Sun, "Double barrier option under regime-switching exponential mean-reverting process," International Journal of Computer Mathematics, vol. 86, no. 6, pp. 964-981, 2009.

[7] X. Guo, "Information and option pricings," Quantitative Finance, vol. 1, no. 1, pp. 38-44, 2001.

[8] X. Guo and Q. Zhang, "Closed-form solutions for perpetual American put options with regime switching," SIAM Journal on Applied Mathematics, vol. 64, no. 6, pp. 2034-2049, 2004.

[9] M. R. Hardy, "A regime-switching model of long-term stock returns," North American Actuarial Journal, vol. 5, no. 2, pp. 4153, 2001.

[10] A. Q. Khaliq and R. H. Liu, "New numerical scheme for pricing American option with regime-switching," International Journal of Theoretical and Applied Finance, vol. 12, no. 3, pp. 319-340, 2009.

[11] A. Q. Khaliq, B. Kleefeld, and R. H. Liu, "Solving complex PDE systems for pricing American options with regime-switching by efficient exponential time differencing schemes," Numerical Methods for Partial Differential Equations, vol. 29, no. 1, pp. 320336, 2013.

[12] R. H. Liu, "Regime-switching recombining tree for option pricing," International Journal of Theoretical and Applied Finance, vol. 13, no. 3, pp. 479-499, 2010.

[13] R. H. Liu and J. L. Zhao, "A lattice method for option pricing with two underlying assets in the regime-switching model," Journal of Computational and Applied Mathematics, vol. 250, pp. 96-106, 2013.

[14] D. D. Yao, Q. Zhang, and X. Y. Zhou, "A regime-switching model for European options," in Stochastic processes, optimization, and control theory: applications in financial engineering, queueing networks, and manufacturing systems, H. M. Yan, G. Yin, and Q. Zhang, Eds., pp. 281-300, Springer, New York, NY, USA, 2006.
[15] F. L. Yuen and H. Yang, "Option pricing with regime switching by trinomial tree method," Journal of Computational and Applied Mathematics, vol. 233, no. 8, pp. 1821-1833, 2010.

[16] F. L. Yuen and H. Yang, "Pricing Asian options and equityindexed annuities with regime switching by the trinomial tree method," North American Actuarial Journal, vol. 14, no. 2, pp. 256-277, 2010.

[17] J. Ma and T. Zhu, "Convergence rates of trinomial tree methods for option pricing under regime-switching models," Applied Mathematics Letters. An International Journal of Rapid Publication, vol. 39, pp. 13-18, 2015.

[18] R. Bansal and H. Zhou, "Term structure of interest rates with regime shifts," Journal of Finance, vol. 57, no. 5, pp. 1997-2043, 2002.

[19] C. Landén, "Bond pricing in a hidden Markov model of the short rate," Finance and Stochastics, vol. 4, no. 4, pp. 371-389, 2000.

[20] R. H. Liu, "A new tree method for pricing financial derivatives in a regime-switching mean-reverting model," Nonlinear Analysis. Real World Applications. An International Multidisciplinary Journal, vol. 13, no. 6, pp. 2609-2621, 2012.

[21] X. Y. Zhou and G. Yin, "Markowitz's mean-variance portfolio selection with regime switching: a continuous-time model," SIAM Journal on Control and Optimization, vol. 42, no. 4, pp. 1466-1482, 2003.

[22] P. Eloe, R. H. Liu, M. Yatsuki, G. Yin, and Q. Zhang, "Optimal selling rules in a regime-switching exponential Gaussian diffusion model," SIAM Journal on Applied Mathematics, vol. 69, no. 3, pp. 810-829, 2008.

[23] G. Yin, R. H. Liu, and Q. Zhang, "Recursive algorithms for stock liquidation: a stochastic optimization approach," SIAM Journal on Optimization, vol. 13, no. 1, pp. 240-263, 2002.

[24] G. Yin, Q. Zhang, F. Liu, R. H. Liu, and Y. Cheng, "Stock liquidation via stochastic approximation using NASDAQ daily and intra-day data," Mathematical Finance, vol. 16, no. 1, pp. 217236, 2006.

[25] Q. Zhang, "Stock trading: an optimal selling rule," SIAM Journal on Control and Optimization, vol. 40, no. 1, pp. 64-87, 2001.

[26] Q. Zhang, G. Yin, and R. H. Liu, "A near-optimal selling rule for a two-time-scale market model," Multiscale Modeling \& Simulation. A SIAM Interdisciplinary Journal, vol. 4, no. 1, pp. 172-193, 2005.

[27] R. Mallier and G. Alobaidi, "Laplace transforms and American options," Applied Mathematical Finance, vol. 7, no. 4, pp. 241256, 2000.

[28] S.-P. Zhu, "A new analytical approximation formula for the optimal exercise boundary of American put options," International Journal of Theoretical and Applied Finance, vol. 9, no. 7, pp. 11411177, 2006.

[29] S.-P. Zhu and J. Zhang, "Using Laplace transform to price American puts," Dynamics of Continuous, Discrete \& Impulsive Systems. Series B. Applications \& Algorithms, vol. 19, no. 4-5, pp. 447-469, 2012.

[30] H. Y. Wong and J. Zhao, "Valuing American options under the CEV model by Laplace-Carson transforms," Operations Research Letters, vol. 38, no. 5, pp. 474-481, 2010.

[31] W. Chen, X. Xu, and S.-P. Zhu, "A predictor-corrector approach for pricing American options under the finite moment logstable model," Applied Numerical Mathematics. An IMACS Journal, vol. 97, pp. 15-29, 2015. 
[32] J.-R. Liang, J. Wang, W.-J. Zhang, W.-Y. Qiu, and F.-Y. Ren, “The solutions to a bi-fractional Black-Scholes-Merton differential equation," International Journal of Pure and Applied Mathematics, vol. 58, no. 1, pp. 99-112, 2010.

[33] W. Chen, X. Xu, and S.-P. Zhu, "Analytically pricing double barrier options based on a time-fractional Black-Scholes equation," Computers \& Mathematics with Applications. An International Journal, vol. 69, no. 12, pp. 1407-1419, 2015.

[34] Z. Zhou and X. Gao, "Numerical methods for pricing American options with time-fractional PDE models," Mathematical Problems in Engineering, vol. 2016, Article ID 5614950, 8 pages, 2016.

[35] I. Podlubny, Fractional Differential Equations, Academic Press, San Diego, Calif, USA, 1999.

[36] P. P. Valkó and J. Abate, "Comparison of sequence accelerators for the Gaver method of numerical Laplace transform inversion," Computers \& Mathematics with Applications, vol. 48, no. 3-4, pp. 629-636, 2004. 


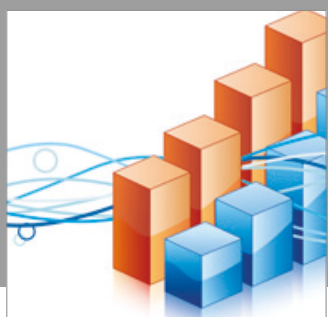

Advances in

Operations Research

vatersals

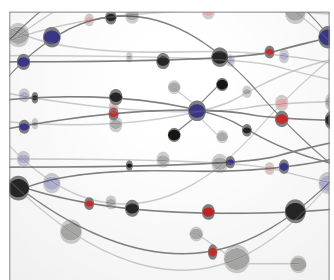

\section{The Scientific} World Journal
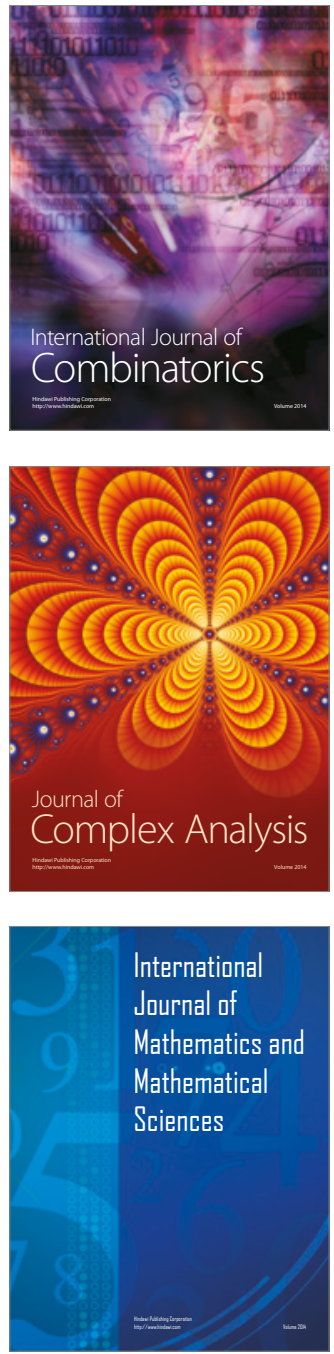
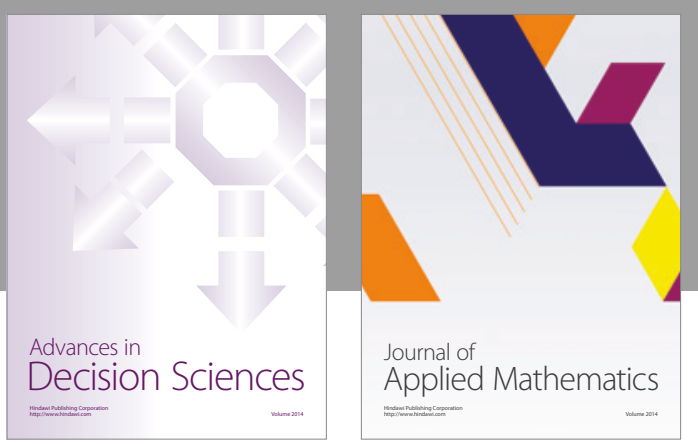

Algebra

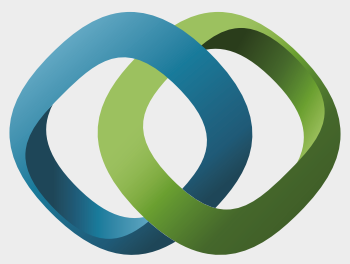

\section{Hindawi}

Submit your manuscripts at

https://www.hindawi.com
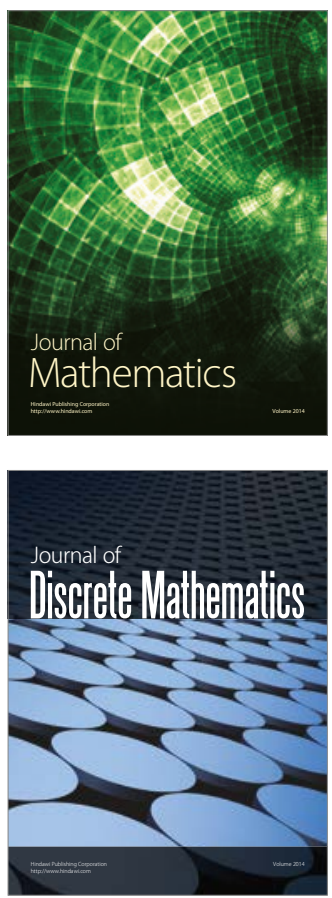

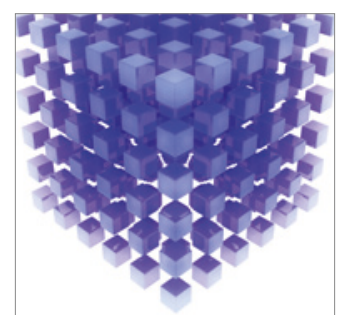

Mathematical Problems in Engineering
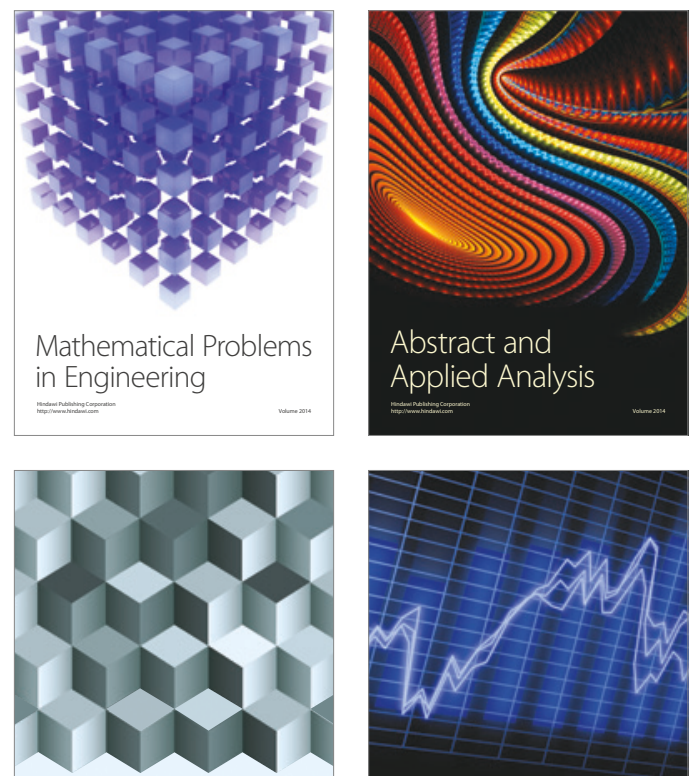

Journal of

Function Spaces

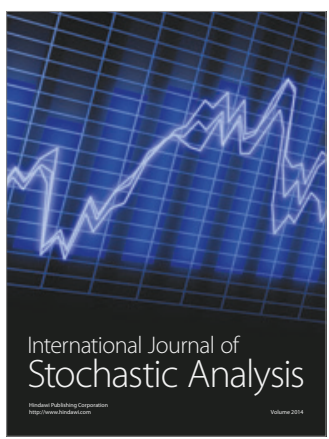

Probability and Statistics
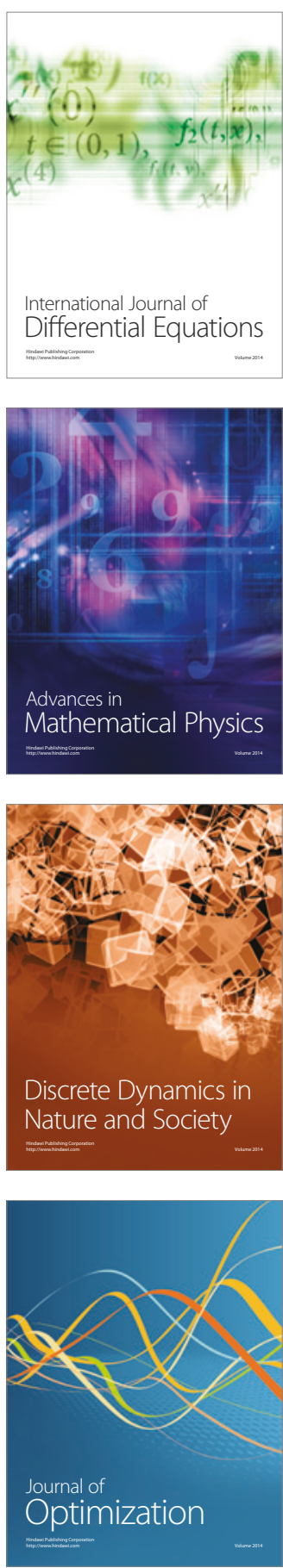